\title{
What should each of us do now?
}

\author{
Toshiko Hirai
}

(C) The Japan Society of Ultrasonics in Medicine 2011

On 11 March 2011, Japan was hit by a massive earthquake, the likes of which are said to occur only once every 1,000 years. Many precious lives were lost due to the resulting tsunami, and not only the daily lives of the people but industry and the economy were also hit hard. Japan has received messages of condolence and encouragement from around the world, and the people of Japan are uniting to overcome this difficulty, with each person doing what he or she can to restore Japan to its former state.

The theme of The 84th Annual Scientific Meeting of the Japan Society of Ultrasonics in Medicine-which is scheduled to be held 27-29 May in Tokyo with Dr. Takenaka as president-is collaboration between the engineers who build the tools for diagnosis and treatment, such as ultrasound devices (engineering), the technologists who actually use the tools (technology), and the doctors who help patients (medicine). Until about 10 years ago, the Japan Society of Ultrasonics in Medicine was proudly characterized by its collaboration between doctors and engineers. Remarkable advances have been made in ultrasound devices in recent years, with many new functions being added, and technologists with a mastery of the devices are becoming instrumental from the standpoint of fully utilizing their capabilities. As such, there seems to be increased attention on the importance of collaboration between doctors, engineers, and now also technologists.

Doctors want to know the important information yielded by ultrasonography, but they do not have the time to thoroughly perform examinations or learn the techniques

\section{T. Hirai $(\square)$}

Central Endoscopy and Ultrasonography Division,

Nara Medical University, Nara Medical University Hospital, Kashihara 634-8522, Japan

e-mail: thirai@naramed-u.ac.jp required to master the devices. Without collaboration between doctors and technologists, technologists would not be able to make use of their techniques because they would not know what kind of information is necessary. Engineers would not be able to build devices with functions that are truly clinically useful if they collaborated only with doctors or only with technologists.

Against this backdrop, examinations are being standardized and guidelines are being generated in each field in recent years. The fact that examinations will yield the minimum required information and that they will differ little no matter where in Japan they are performed are positives from the standpoint of improving the objectivity of ultrasonography and being able to discuss ultrasonographic findings on an equal footing. When standards and guidelines are generated, however, people tend to focus only on performing examinations that comply totally with the standards and guidelines and stop thinking on their own. When the ultrasound examination request or complaint of the patient do not coincide with the ultrasonographic findings, it is vital to think about why the examination yielded such findings, and to consider adding parameters not in the guidelines and how the findings will benefit the subsequent diagnosis and treatment. Further advances in medical ultrasonics and the important process for obtaining new findings must not be lost due to standardization and generation of guidelines.

What should we do? Feeling that it is important for doctors, engineers, and technologists to have a venue to get together and talk, Dr. Takenaka has organized many special programs for collaboration between the three parties. If the doctors indicate that it would be beneficial to have certain information revealed by ultrasound, the technologists might respond that current ultrasound technology cannot yield that information but could provide some other 
similar information, and the engineers might suggest the technology necessary to make it happen. Everyone works together as a team. If such relationships can be formed, it might be possible to overcome the various difficult problems facing us today. To that end, doctors must think about how to improve medical care, technologists must think about what information ultrasound can yield and how accurate it is, and engineers must think about what needs to be done to create the devices needed in clinical settings.

I think the slogan for overcoming the unprecedented Great Eastern Japan Earthquake-i.e., uniting to overcome this difficulty, with each person doing what he or she canis exactly the same slogan that is needed for the future advancement of medical ultrasonics. 